\title{
Approximation of the inverse elliptic problem with mixed boundary value conditions and overdetermination
}

\author{
Charyyar Ashyralyyev ${ }^{1,2}$ and Mutlu Dedeturk ${ }^{1 *}$
}

\section{*Correspondence: \\ mutludedeturk@gumushane.edu.tr 'Department of Mathematical Engineering, Gumushane University, Gumushane, 29100, Turkey Full list of author information is available at the end of the article}

\begin{abstract}
In the present study, the inverse problem for a multidimensional elliptic equation with mixed boundary conditions and overdetermination is considered. The first and second orders of accuracy in $t$ and the second order of accuracy in space variables for the approximate solution of this inverse problem are constructed. Stability, almost coercive stability, and coercive stability estimates for the solution of these difference schemes are established. For the two-dimensional inverse problems with mixed boundary value conditions, numerical results are presented in test examples.
\end{abstract}

MSC: 35N25; 39A14; 39A30; 65J22

Keywords: overdetermination; approximation; inverse elliptic problem; multidimensional elliptic equation; well-posedness; stability estimate; almost coercive stability; coercive stability

\section{Introduction}

Inverse problems for partial differential equations are frequently encountered in various branches of science (see [1-3] and the bibliography therein).

In recent years, theory and methods of solutions of inverse problems of determining the parameter of partial differential equations have been extensively studied by several researchers (see [1-25] and the references therein).

We will denote the set of natural numbers from 1 to $n$ by $\mathbb{N}_{n}=\{1,2, \ldots, n\}$, and closure of set $\Omega$ by $\bar{\Omega}=\Omega \cup S$.

Let $n \in \mathbb{N}, \delta, T, L>0, \lambda \in(0, T)$ be given numbers and $\Omega=(0, L) \times \cdots \times(0, L)$ be the open cube in the $n$-dimensional Euclidean space with boundary $S=\partial \Omega=S_{1} \cup S_{2}$, where

$$
\begin{aligned}
& S=\bigcup_{i=1}^{n}\left\{x=\left(x_{1}, x_{2}, \ldots x_{n}\right) \mid x_{i}=0 \text { or } x_{i}=L, x_{k} \in[0, L], k \in \mathbb{N}_{n} \backslash\{i\}\right\}, \\
& S_{1}=\bigcup_{i=1}^{n}\left\{x=\left(x_{1}, x_{2}, \ldots x_{n}\right) \mid x_{i}=0, x_{k} \in[0, L], k \in \mathbb{N}_{n} \backslash\{i\}\right\}, \\
& S_{2}=\bigcup_{i=1}^{n}\left\{x=\left(x_{1}, x_{2}, \ldots x_{n}\right) \mid x_{i}=L, x_{k} \in(0, L], k \in \mathbb{N}_{n} \backslash\{i\}\right\},
\end{aligned}
$$

\section{Springer}

(0) 2015 Ashyralyyev and Dedeturk; licensee Springer. This is an Open Access article distributed under the terms of the Creative Commons Attribution License (http://creativecommons.org/licenses/by/4.0), which permits unrestricted use, distribution, and reproduction in any medium, provided the original work is properly credited. 
and let $a_{r}, \varphi, \xi, \psi: \bar{\Omega} \rightarrow \mathbb{R}, f:(0, T) \times \Omega \rightarrow \mathbb{R}$ be given smooth functions such that $a_{r}(x) \geq$ $a>0$ for $x \in \bar{\Omega}, r \in \mathbb{N}_{n}$. Consider the multidimensional elliptic equation

$$
\begin{gathered}
-u_{t t}(t, x)-\sum_{r=1}^{n}\left(a_{r}(x) u_{x_{r}}(t, x)\right)_{x_{r}}+\delta u(t, x)=f(t, x)+p(x), \\
x=\left(x_{1}, \ldots, x_{n}\right) \in \Omega, 0<t<T .
\end{gathered}
$$

Here $u(t, x)$ and $p(x)$ are unknown functions.

In $[0, T] \times \Omega$, consider the inverse problem of finding functions $u(t, x)$ and $p(x)$ for the multidimensional elliptic equation (1.1) with overdetermined boundary conditions

$$
u(0, x)=\varphi(x), \quad u(\lambda, x)=\xi(x), \quad u(T, x)=\psi(x), \quad x \in \bar{\Omega}
$$

and one of the following usual boundary conditions on $[0, T] \times S$ :

$$
\begin{aligned}
& u(t, x)=0, \quad 0 \leq t \leq T, x \in S, \\
& \frac{\partial u(t, x)}{\partial \vec{n}}=0, \quad 0 \leq t \leq T, x \in S, \\
& \frac{\partial u(t, x)}{\partial \vec{n}}=0, \quad x \in S_{1}, \quad u(t, x)=0, \quad x \in S_{2}, 0 \leq t \leq T, \\
& u(t, x)=0, \quad x \in S_{1}, \quad \frac{\partial u(t, x)}{\partial \vec{n}}=0, \quad x \in S_{2}, 0 \leq t \leq T .
\end{aligned}
$$

For the problem of determining the parameter of an elliptic equation in a Banach space, the exact estimates of solution in Hölder norms are established in [21]. Well-posedness of inverse problem (1.1), (1.2), (1.3) and its approximations were investigated in [18]. In [21], the third and fourth order stable difference schemes for approximate solution of this problem were presented. Stability and coercive stability estimates for the solution of the first and second order difference schemes for inverse problem (1.1), (1.2), (1.4) were established in [19]. In [23], the authors studied inverse problem (1.1), (1.2), (1.5).

Our aim in this work is to construct the first and second orders of accuracy difference schemes for an approximate solution of inverse problem (1.1), (1.2), (1.6) and to establish stability, coercive stability, almost coercive stability estimates for the solution of these difference schemes.

This paper is planned as follows. In Section 2, we give theorems on well-posedness of inverse problems with mixed boundary conditions and overdetermination. Section 3 is devoted to the construction of the first and second order difference schemes for approximate solution of problem (1.1), (1.2), (1.6). In this section, we establish stability, almost coercive, and coercive stability inequalities for the solution of difference schemes. In Section 4, we present numerical results for a two-dimensional elliptic equation. The conclusion is given in the final Section 5 .

\section{Well-posedness of inverse problems with mixed boundary conditions and overdetermination}

Let $\mathcal{C}_{0 T}^{\alpha, \alpha}\left(L_{2}(\bar{\Omega})\right)$ be the space obtained by completion of the space of all smooth $L_{2}(\bar{\Omega})$ valued functions $g$ on $[0, T]$ with the norm

$$
\|g\|_{\mathcal{C}_{0 T}^{\alpha, \alpha}\left(L_{2}(\bar{\Omega})\right)}=\|g\|_{\mathcal{C}\left(L_{2}(\bar{\Omega})\right)}+\sup _{0 \leq t<t+\tau \leq T} \frac{(t+\tau)^{\alpha}(T-t)^{\alpha}\|g(t+\tau)-g(t)\|_{L_{2}(\bar{\Omega})}}{\tau^{\alpha}} .
$$


Denote the differential expression generated by (1.1) [26]

$$
A \rho(x)=-\sum_{r=1}^{n}\left(a_{r}(x) \rho_{x_{r}}(x)\right)_{x_{r}}+\delta \rho(x)
$$

where $\rho \in W_{2}^{2}(\bar{\Omega})$.

The differential expression (1.1) defines a self-adjoint positive definite operator $A$ acting on $L_{2}(\bar{\Omega})$ with the domain

$$
D(A)=\left\{\rho \in W_{2}^{2}(\bar{\Omega}), \frac{\partial \rho}{\partial \vec{n}}=0 \text { on } S_{1}, \rho=0 \text { on } S_{2}\right\} .
$$

Therefore, by using abstract Theorems 2.1 and 2.2 of paper [19] for $H=L_{2}(\bar{\Omega})$, we get the following theorems on well-posedness of inverse problem (1.1), (1.2), (1.5).

Theorem 2.1 Suppose that $A$ is defined by formula (2.1), $\varphi, \xi, \psi \in D(A)$. Then, for the solutions $(u, p)$ of inverse problem (1.1), (1.2), (1.5), the stability inequalities

$$
\begin{aligned}
&\|u\|_{C\left(L_{2}(\bar{\Omega})\right)} \leq M_{1}\left[\|\varphi\|_{L_{2}(\bar{\Omega})}+\|\psi\|_{L_{2}(\bar{\Omega})}+\|\xi\|_{L_{2}(\bar{\Omega})}+\|f\|_{C\left(L_{2}(\bar{\Omega})\right)}\right] \\
&\left\|A^{-1} p\right\|_{L_{2}(\bar{\Omega})} \leq M_{1}\left[\|\varphi\|_{L_{2}(\bar{\Omega})}+\|\psi\|_{L_{2}(\bar{\Omega})}+\|\xi\|_{L_{2}(\bar{\Omega})}+\|f\|_{C\left(L_{2}(\bar{\Omega})\right)}\right] \\
&\|p\|_{L_{2}(\bar{\Omega})} \leq M_{1}\left[\|A \varphi\|_{L_{2}(\bar{\Omega})}+\|A \psi\|_{L_{2}(\bar{\Omega})}+\|A \xi\|_{L_{2}(\bar{\Omega})}\right. \\
&\left.+\frac{1}{\alpha(1-\alpha)}\|f\|_{\mathcal{C}_{0 T}^{\alpha, \alpha}\left(L_{2}(\bar{\Omega})\right)}\right]
\end{aligned}
$$

are satisfied, where $M_{1}$ is independent of $\alpha, \varphi(x), \xi(x), \psi(x)$, and $f(t, x)$.

Theorem 2.2 Assume that $A$ is defined by formula (2.1), $\varphi, \psi, \xi \in D(A)$. Then, for the solution of inverse problem (1.1), (1.2), (1.5), the coercive stability estimate

$$
\begin{aligned}
& \left\|u^{\prime \prime}\right\|_{\mathcal{C}_{0 T}^{\alpha, \alpha}\left(L_{2}(\bar{\Omega})\right)}+\|u\|_{\mathcal{C}_{0 T}^{\alpha, \alpha}\left(W_{2}^{2}(\bar{\Omega})\right)}+\|p\|_{L_{2}(\bar{\Omega})} \\
& \quad \leq M_{2}\left[\frac{1}{\alpha(1-\alpha)}\|f\|_{\mathcal{C}_{0 T}^{\alpha, \alpha}\left(L_{2}(\bar{\Omega})\right)}+\|\varphi\|_{W_{2}^{2}(\bar{\Omega})}+\|\psi\|_{W_{2}^{2}(\bar{\Omega})}+\|\xi\|_{W_{2}^{2}(\bar{\Omega})}\right]
\end{aligned}
$$

holds, where $M_{2}$ does not depend on $\alpha, \varphi(x), \xi(x), \psi(x)$, and $f(t, x)$.

Now, consider inverse problem (1.1), (1.2), (1.6).

The differential expression (1.1) defines a self-adjoint positive definite operator $A$ acting on $L_{2}(\bar{\Omega})$ with the domain

$$
D(A)=\left\{\rho \in W_{2}^{2}(\bar{\Omega}), \rho=0 \text { on } S_{1}, \frac{\partial \rho}{\partial \vec{n}}=0 \text { on } S_{2}\right\} .
$$

So, in a similar manner, applying abstract Theorems 2.1 and 2.2 of paper [19] for $H=$ $L_{2}(\bar{\Omega})$, we can get the theorems on well-posedness of inverse problem (1.1), (1.2), (1.6). 
Theorem 2.3 Suppose that $A$ is defined by formula (2.1), $\varphi, \xi, \psi \in D(A)$. Then, for the solution (u, p) of inverse problem (1.1), (1.2), (1.6), the stability inequalities (2.2), (2.3), (2.4) hold.

Theorem 2.4 Assume that $A$ is defined by formula (2.1), $\varphi, \psi, \xi \in D(A)$. Then, for the solution $(u, p)$ of inverse problem (1.1), (1.2), (1.6), coercive stability estimate (2.5) is valid.

\section{Difference schemes and their well-posedness}

We discretize inverse problem (1.1), (1.2), (1.6) in two steps. In the first step, define the set of grid points in space variables,

$$
\left\{\begin{array}{l}
\widetilde{\Omega}_{h}=\left\{x=\left(h_{1} m_{1}, \ldots, h_{h} m_{h}\right) \mid m_{i}=0, \ldots, M_{i}, h_{i} M_{i}=L, i=1, \ldots, n\right\}, \\
\Omega_{h}=\widetilde{\Omega}_{h} \cap \Omega, \quad S_{h}^{1}=\widetilde{\Omega}_{h} \cap S^{1}, \quad S_{h}^{2}=\widetilde{\Omega}_{h} \cap S^{2} .
\end{array}\right.
$$

Introduce the Hilbert spaces $L_{2 h}=L_{2}\left(\widetilde{\Omega}_{h}\right)$ and $W_{2 h}^{2}=W_{2}^{2}\left(\widetilde{\Omega}_{h}\right)$ of grid functions $g^{h}(x)=$ $\left\{g\left(h_{1} m_{1}, \ldots, h_{n} m_{n}\right) \mid m_{i}=0, \ldots, M_{i}, i=1, \ldots, n\right\}$ defined on $\widetilde{\Omega}_{h}$ equipped with the norms

$$
\begin{aligned}
\left\|g^{h}\right\|_{L_{2 h}}= & \left(\sum_{x \in \widetilde{\Omega}_{h}}\left|g^{h}(x)\right|^{2} h_{1} \cdots h_{n}\right)^{1 / 2}, \\
\left\|g^{h}\right\|_{W_{2 h}^{2}=} & \left\|g^{h}\right\|_{L_{2 h}}+\left(\sum_{x \in \tilde{\Omega}_{h}} \sum_{i=1}^{n}\left|\left(g^{h}(x)\right)_{x_{i}, m_{i}}\right|^{2} h_{1} \cdots h_{h}\right)^{1 / 2} \\
& +\left(\sum_{x \in \tilde{\Omega}_{h}} \sum_{i=1}^{n}\left|\left(g^{h}(x)\right)_{x_{i} \bar{x}_{i}, m_{i}}\right|^{2} h_{1} \cdots h_{h}\right)^{1 / 2},
\end{aligned}
$$

respectively.

To the differential operator $A(2.1)$ assign the difference operator $A_{h}^{x}$, defined by the formula

$$
A_{h}^{x} u^{h}=-\sum_{i=1}^{n}\left(a_{i}(x) u_{\bar{x}_{i}}^{h}\right)_{x_{i}, m_{i}}+\delta u^{h}
$$

acting in the space of grid functions $u^{h}(x)$ satisfying the conditions $u^{h}(x)=0$ for all $x \in S_{h}^{1}$ and $D^{h} u^{h}(x)=0$ for all $x \in S_{h}^{2}$. Here, $D^{h} u^{h}(x)$ is an approximation of $\frac{\partial u}{\partial \breve{n}}$. Note that $[26,27]$ $A_{h}^{x}$ is a self-adjoint positive definite operator in $L_{2}\left(\widetilde{\Omega}_{h}\right)$.

In this step, by using $A_{h}^{x}$, for obtaining $u^{h}(t, x)$ functions, we arrive at the problem

$$
\begin{cases}-\frac{d^{2} u^{h}(t, x)}{d t^{2}}+A_{h}^{x} u^{h}(t, x)=f^{h}(t, x)+p^{h}(x), & 0<t<T, x \in \Omega_{h}, \\ u^{h}(0, x)=\varphi^{h}(x), \quad u^{h}(\lambda, x)=\xi^{h}(x), & u^{h}(T, x)=\psi^{h}(x), \quad x \in \widetilde{\Omega}_{h} .\end{cases}
$$

In the second step, applying the approximate formula

$$
u^{h}(\lambda, x)=u^{h}\left(\left[\frac{\lambda}{\tau}\right] \tau, x\right)+o(\tau)
$$


for $u^{h}(\lambda, x)=\xi^{h}(x)$, we replace inverse problem (1.1), (1.2), (1.6) with the first order of accuracy difference scheme

$$
\left\{\begin{array}{l}
-\frac{u_{k+1}^{h}(x)-2 u_{k}^{h}(x)+u_{k-1}^{h}(x)}{\tau^{2}}+A_{h}^{x} u_{k}^{h}(x)=\theta_{k}^{h}(x)+p^{h}(x), \\
\theta_{k}^{h}(x)=f^{h}\left(t_{k}, x\right), t_{k}=k \tau, 1 \leq k \leq N-1, x \in \Omega_{h}, \\
u_{0}^{h}(x)=\varphi^{h}(x), \quad u_{N}^{h}(x)=\psi^{h}(x), \quad u_{l}^{h}(x)=\xi^{h}(x), \quad x \in \widetilde{\Omega}_{h}, N \tau=T .
\end{array}\right.
$$

Here, $l=\left[\frac{\lambda}{\tau}\right],[\cdot]$ is a notation for the greatest integer function.

In this step, by using the approximate formula

$$
u^{h}(\lambda, x)=u^{h}(l \tau, x)+\left(\frac{\lambda}{\tau}-l\right)\left(u^{h}(l \tau+\tau, x)-u^{h}(l \tau, x)\right)+o\left(\tau^{2}\right)
$$

for $u^{h}(\lambda, x)=\xi^{h}(x)$, we construct the second order of accuracy difference scheme for inverse problem (1.1), (1.2), (1.6)

$$
\left\{\begin{array}{l}
-\frac{u_{k+1}^{h}(x)-2 u_{k}^{h}(x)+u_{k-1}^{h}(x)}{\tau^{2}}+A_{h}^{x} u_{k}^{h}(x)=\theta_{k}^{h}(x)+p^{h}(x), \\
\theta_{k}^{h}(x)=f^{h}\left(t_{k}, x\right), t_{k}=k \tau, 1 \leq k \leq N-1, x \in \widetilde{\Omega}_{h}, \\
u_{0}^{h}(x)=\varphi^{h}(x), \quad u_{N}^{h}(x)=\psi^{h}(x), \\
u_{l}^{h}(x)+\left(\frac{\lambda}{\tau}-l\right)\left(u_{l+1}^{h}(x)-u_{l}^{h}(x)\right)=\xi^{h}(x), \quad x \in \widetilde{\Omega}_{h}, N \tau=T .
\end{array}\right.
$$

Applying

$$
u_{k}^{h}(x)=v_{k}^{h}(x)-\left(A_{h}^{x}\right)^{-1} p^{h}(x), \quad 1 \leq k \leq N-1,
$$

we can reduce difference problems (3.3) and (3.4) to the auxiliary nonlocal difference problems

$$
\left\{\begin{array}{l}
-\frac{v_{k+1}^{h}(x)-2 v_{k}^{h}(x)+v_{k-1}^{h}(x)}{\tau^{2}}+A_{h}^{x} v_{k}^{h}(x)=\theta_{k}^{h}(x), \\
\quad \theta_{k}^{h}(x)=f^{h}\left(t_{k}, x\right), t_{k}=k \tau, 1 \leq k \leq N-1, x \in \Omega_{h}, \\
v_{0}^{h}(x)-v_{l}^{h}(x)=\varphi^{h}(x)-\xi^{h}(x), \\
v_{N}^{h}(x)-v_{l}^{h}(x)=\psi^{h}(x)-\xi^{h}(x), \quad x \in \widetilde{\Omega}_{h}, N \tau=T
\end{array}\right.
$$

and

$$
\left\{\begin{array}{l}
-\frac{v_{k+1}^{h}(x)-2 v_{k}^{h}(x)+v_{k-1}^{h}(x)}{\tau^{2}}+A_{h}^{x} v_{k}^{h}(x)=\theta_{k}^{h}(x), \\
\theta_{k}^{h}(x)=f^{h}\left(t_{k}, x\right), t_{k}=k \tau, 1 \leq k \leq N-1, x \in \widetilde{\Omega}_{h}, \\
v_{0}^{h}(x)-v_{l}^{h}(x)-\left(\frac{\lambda}{\tau}-l\right)\left(v_{l+1}^{h}(x)-v_{l}^{h}(x)\right)=\varphi^{h}(x)-\xi^{h}(x), \\
v_{N}^{h}(x)-v_{l}^{h}(x)-\left(\frac{\lambda}{\tau}-l\right)\left(v_{l+1}^{h}(x)-v_{l}^{h}(x)\right)=\psi^{h}(x)-\xi^{h}(x), \\
\quad x \in \widetilde{\Omega}_{h}, N \tau=T,
\end{array}\right.
$$

respectively.

The difference schemes for nonlocal boundary value problems for the multidimensional elliptic equation were studied in $[28,29]$. 
Denote

$$
C=\frac{1}{2}\left(\tau A_{h}^{x}+\sqrt{4 A_{h}^{x}+\tau^{2}\left(A_{h}^{x}\right)^{2}}\right), \quad R=(I+\tau C)^{-1} .
$$

Let $H$ be the Hilbert space $L_{2}(\bar{\Omega})$.

Lemma 3.1 The following estimates are satisfied [27]:

$$
\begin{aligned}
& \left\|R^{k}\right\|_{H \rightarrow H} \leq M_{3}\left(1+\delta^{\frac{1}{2}} \tau\right)^{-k}, \quad \delta>0 \\
& \left\|C R^{k}\right\|_{H \rightarrow H} \leq \frac{M_{3}}{k \tau}, \quad k \geq 1 \\
& \left\|\left(I-R^{2 N}\right)^{-1}\right\|_{H \rightarrow H} \leq M_{3}
\end{aligned}
$$

where $M_{3}$ is independent of $\tau$.

Lemma 3.2 ([19]) For $1 \leq l \leq N-1$ and for the operator $S=R^{2 N}+R^{l}-R^{2 N-l}+R^{N-l}-R^{N+l}$, the operator $I-S$ has an inverse $G=(I-S)^{-1}$ and the estimate

$$
\|G\|_{H \rightarrow H} \leq M_{4}
$$

is satisfied, where $M_{4}$ does not depend on $\tau$.

Lemma 3.3 ([19]) For $1 \leq l \leq N-1$ and for the operator

$$
\begin{aligned}
S_{1}= & R^{2 N}-\left(\frac{\lambda}{\tau}-l-1\right)\left(R^{l}-R^{2 N-l}+R^{N-l}-R^{N+l}\right) \\
& +\left(\frac{\lambda}{\tau}-l\right)\left(R^{l+1}-R^{2 N-l-1}+R^{N-l-1}-R^{N+l+1}\right),
\end{aligned}
$$

the operator $I-S_{1}$ has an inverse

$$
\begin{aligned}
G_{1}= & \left(I-R^{2 N}+\left(\frac{\lambda}{\tau}-l-1\right)\left(R^{l}-R^{2 N-l}+R^{N-l}-R^{N+l}\right)\right. \\
& \left.-\left(\frac{\lambda}{\tau}-l\right)\left(R^{l+1}-R^{2 N-l-1}+R^{N-l-1}-R^{N+l+1}\right)\right)^{-1},
\end{aligned}
$$

and the estimate

$$
\left\|G_{1}\right\|_{H \rightarrow H} \leq M_{5}
$$

is valid, where $M_{5}$ does not depend on $\tau$.

In a similar manner as [19], we can obtain the following representation formula

$$
\begin{aligned}
u_{k}^{h}(x)= & \left(I-R^{2 N}\right)^{-1}\left[\left(\left(R^{k}-R^{2 N-k}\right) v_{0}^{h}(x)+\left(R^{N-k}-R^{N+k}\right) v_{N}^{h}(x)\right)\right. \\
& \left.-\left(R^{N-k}-R^{N+k}\right)(I+\tau C)(2 I+\tau C)^{-1} C^{-1} \sum_{i=1}^{N-1}\left(R^{N-i}-R^{N+i}\right) f_{i}^{h}(x) \tau\right]
\end{aligned}
$$




$$
\begin{aligned}
& +(I+\tau C)(2 I+\tau C)^{-1} C^{-1} \sum_{i=1}^{N-1}\left(R^{|k-i|}-R^{k+i}\right) f_{i}^{h}(x) \tau+\varphi^{h}(x)-v_{0}^{h}(x), \\
p^{h}(x)= & A_{h}^{x} \varphi^{h}(x)-A_{h}^{x} v_{0}^{h}(x), \\
v_{N}^{h}(x)= & v_{0}^{h}(x)+\psi^{h}(x)-\varphi^{h}(x),
\end{aligned}
$$

with

$$
\begin{aligned}
v_{0}^{h}(x)= & -G\left(R^{N-l}-R^{N+l}\right)(I+\tau C)(2 I+\tau C)^{-1} C^{-1} \\
& \times \sum_{i=1}^{N-1}\left(R^{N-i}-R^{N+i}\right) f_{i}^{h}(x) \tau+G\left(I-R^{2 N}\right)(I+\tau C) \\
& \times(2 I+\tau C)^{-1} C^{-1} \sum_{i=1}^{N-1}\left(R^{|l-i|}-R^{l+i}\right) f_{i}^{h}(x) \tau \\
& +G\left(I-R^{2 N}\right)\left(\varphi^{h}(x)-\xi^{h}(x)\right)+G\left(R^{N-l}-R^{N+l}\right)\left(\psi^{h}(x)-\varphi^{h}(x)\right)
\end{aligned}
$$

for the solution of difference scheme (3.3) and

$$
\begin{aligned}
v_{0}^{h}(x)= & \left(\frac{\lambda}{\tau}-l-1\right) G_{1}\left(R^{N-l}-R^{N+l}\right)(I+\tau C)(2 I+\tau C)^{-1} C^{-1} \\
& \times \sum_{i=1}^{N-1}\left(R^{N-i}-R^{N+i}\right) f_{i}^{h}(x) \tau \\
& -\left(\frac{\lambda}{\tau}-l-1\right) G_{1}\left(I-R^{2 N}\right)(I+\tau C)(2 I+\tau C)^{-1} C^{-1} \\
& \times \sum_{i=1}^{N-1}\left(R^{|l-i|}-R^{l+i}\right) f_{i}^{h}(x) \tau \\
& -\left(\frac{\lambda}{\tau}-l\right) G_{1}\left(R^{N-l-1}-R^{N+l+1}\right)(I+\tau C)(2 I+\tau C)^{-1} C^{-1} \\
& \times \sum_{i=1}^{N-1}\left(R^{N-i}-R^{N+i}\right) f_{i}^{h}(x) \tau \\
& +\left(\frac{\lambda}{\tau}-l\right) G_{1}\left(I-R^{2 N}\right)(I+\tau C)(2 I+\tau C)^{-1} C^{-1} \\
& \times \sum_{i=1}^{N-1}\left(R^{|l+1-i|}-R^{l+1+i}\right) f_{i}^{h}(x) \tau \\
& +G_{1}\left(I-R^{2 N}\right)\left(\varphi^{h}(x)-\xi^{h}(x)\right)+\left(\left(\frac{\lambda}{\tau}-l-1\right) G_{1}\left(R^{N-l}-R^{N+l}\right)\right. \\
& \left.+\left(\frac{\lambda}{\tau}-l\right) G_{1}\left(R^{N-l-1}-R^{N+l+1}\right)\right)\left(\psi^{h}(x)-\varphi^{h}(x)\right)
\end{aligned}
$$

for difference scheme (3.4).

Now, we consider the linear spaces of mesh functions $\theta^{\tau}=\left\{\theta_{k}\right\}_{1}^{N-1}$ with values in the Hilbert space $H$. We denote by $C\left([0, T]_{\tau}, H\right)$ a normed space with the norm

$$
\left\|\left\{\theta_{k}\right\}_{1}^{N-1}\right\|_{C\left([0, T]_{\tau}, H\right)}=\max _{1 \leq k \leq N-1}\left\|\theta_{k}\right\|_{H},
$$


and by $\mathcal{C}_{0 T}^{\alpha, \alpha}\left([0, T]_{\tau}, H\right)$ a normed space with the norm

$$
\begin{aligned}
\left\|\left\{\theta_{k}\right\}_{1}^{N-1}\right\|_{\mathcal{C}_{0 T}^{\alpha, \alpha}\left([0, T]_{\tau}, H\right)}= & \left\|\left\{\theta_{k}\right\}_{1}^{N-1}\right\|_{C\left([0, T]_{\tau}, H\right)} \\
& +\sup _{1 \leq k<k+n \leq N-1} \frac{(k \tau+n \tau)^{\alpha}(T-k \tau)^{\alpha}\left\|\theta_{k+n}-\theta_{k}\right\|_{H}}{(n \tau)^{\alpha}} .
\end{aligned}
$$

Let $\tau$ and $|h|=\sqrt{h_{1}^{2}+\cdots+h_{n}^{2}}$ be sufficiently small positive numbers.

Theorem 3.1 The solutions $\left(\left\{u_{k}^{h}\right\}_{1}^{N-1}, p^{h}\right)$ of difference schemes (3.3) and (3.4) obey the following stability estimates:

$$
\begin{aligned}
& \left\|\left\{u_{k}^{h}\right\}_{1}^{N-1}\right\|_{C\left([0, T]_{\tau}, L_{2 h}\right)} \leq M_{6}\left[\left\|\varphi^{h}\right\|_{L_{2 h}}+\left\|\psi^{h}\right\|_{L_{2 h}}+\left\|\xi^{h}\right\|_{L_{2 h}}+\left\|\left\{f_{k}^{h}\right\}_{1}^{N-1}\right\|_{C\left([0, T]_{\tau}, L_{2 h}\right)}\right], \\
& \left\|p^{h}\right\|_{L_{2 h}} \leq M_{6}\left[\left\|\varphi^{h}\right\|_{W_{2 h}^{2}}+\left\|\psi^{h}\right\|_{W_{2 h}^{2}}+\left\|\xi^{h}\right\|_{W_{2 h}^{2}}+\frac{1}{\alpha(1-\alpha)}\left\|\left\{f_{k}^{h}\right\}_{1}^{N-1}\right\|_{\mathcal{C}_{0 T}^{\alpha, \alpha}\left([0, T]_{\tau}, L_{2 h}\right)}\right]
\end{aligned}
$$

where $M_{6}$ is independent of $\tau, \alpha, h, \varphi^{h}, \psi^{h}, \xi^{h}$, and $\left\{f_{k}^{h}\right\}_{1}^{N-1}$.

Theorem 3.2 The solutions of difference schemes (3.3) and (3.4) obey the following almost coercive stability estimate:

$$
\begin{aligned}
& \left\|\left\{\frac{u_{k+1}^{h}-2 u_{k}^{h}+u_{k-1}^{h}}{\tau^{2}}\right\}_{1}^{N-1}\right\|_{C\left([0, T]_{\tau}, L_{2 h}\right)}+\left\|p^{h}\right\|_{L_{2 h}} \\
& \quad \leq M_{7}\left(\left\|\varphi^{h}\right\|_{W_{2 h}^{2}}+\left\|\psi^{h}\right\|_{W_{2 h}^{2}}+\left\|\xi^{h}\right\|_{W_{2 h}^{2}}+\ln \left(\frac{1}{\tau+h}\right)\left\|\left\{f_{k}^{h}\right\}_{1}^{N-1}\right\|_{C\left([0, T]_{\tau}, L_{2 h}\right)}\right),
\end{aligned}
$$

where $M_{7}$ does not depend on $\tau, \alpha, h, \varphi^{h}, \psi^{h}, \xi^{h}$, and $\left\{f_{k}^{h}\right\}_{1}^{N-1}$.

Theorem 3.3 The solutions of difference schemes (3.3) and (3.4) obey the following coercive stability estimate:

$$
\begin{aligned}
& \left\|\left\{\frac{u_{k+1}^{h}-2 u_{k}^{h}+u_{k-1}^{h}}{\tau^{2}}\right\}_{1}^{N-1}\right\|_{\mathcal{C}_{0 T}^{\alpha, \alpha}\left([0, T]_{\tau}, L_{2 h}\right)}+\left\|\left\{u_{k}^{h}\right\}_{1}^{N-1}\right\|_{\mathcal{C}_{0 T}^{\alpha, \alpha}\left([0, T]_{\tau}, W_{2 h}^{2}\right)}+\left\|p^{h}\right\|_{L_{2 h}} \\
& \quad \leq M_{8}\left[\frac{1}{\alpha(1-\alpha)}\left\|\left\{f_{k}^{h}\right\}_{1}^{N-1}\right\|_{\mathcal{C}_{0 T}^{\alpha, \alpha}\left([0, T]_{\tau}, L_{2 h}\right)}+\left\|\varphi^{h}\right\|_{W_{2 h}^{2}}+\left\|\psi^{h}\right\|_{W_{2 h}^{2}}+\left\|\xi^{h}\right\|_{W_{2 h}^{2}}\right],
\end{aligned}
$$

where $M_{8}$ is independent of $\tau, \alpha, h, \varphi^{h}, \psi^{h}, \xi^{h}$, and $\left\{f_{k}^{h}\right\}_{1}^{N-1}$.

The proofs of Theorems 3.1-3.3 are based on the symmetry property of operator $A_{h}^{x}$ in $L_{2 h}$, representation formulas (3.10), (3.11), (3.12), Lemmas 3.1-3.3 and the following theorem on the coercivity estimate for the solution of the elliptic difference problem in $L_{2 h}$.

Theorem 3.4 ([30]) For the solution of the elliptic difference problem

$$
\left\{\begin{array}{l}
A_{h}^{x} u^{h}(x)=\omega^{h}(x), \quad x \in \widetilde{\Omega}_{h}, \\
u^{h}(x)=0, \quad x \in S_{1}^{h}, \quad D^{h} u^{h}(x)=0, \quad x \in S_{2}^{h},
\end{array}\right.
$$


the following coercivity inequality holds:

$$
\sum_{q=1}^{n}\left\|\left(u_{k}^{h}\right)_{x_{q} \bar{x}_{q}, m_{q}}\right\|_{L_{2 h}} \leq M\left\|\omega^{h}\right\|_{L_{2 h}},
$$

where $M$ does not depend on $h$ and $\omega^{h}$.

\section{Numerical example}

We consider the inverse problem

$$
\left\{\begin{array}{l}
-\frac{\partial^{2} u(t, x)}{\partial t^{2}}-\frac{\partial}{\partial x}\left((2+\cos x) \frac{\partial u(t, x)}{\partial x}\right)+u(t, x)=f(t, x)+p(x), \\
\quad 0<x<\pi, 0<t<T, \\
f(t, x)=-\exp (-t)(\cos (x)-1)+(\exp (-t)+t)(3 \cos (x)+\cos (2 x)-1), \\
u(0, x)=2(\cos (x)-1), \quad 0 \leq x \leq \pi, \\
u(T, x)=(\exp (-T)+T+1)(\cos (x)-1), \quad 0 \leq x \leq \pi, \\
u(\lambda, x)=(\exp (-\lambda)+\lambda+1)(\cos (x)-1), \quad 0 \leq x \leq \pi, \\
u(t, 0)=u_{x}(t, \pi)=0, \quad 0 \leq t \leq T, \lambda=\frac{4 T}{7}
\end{array}\right.
$$

for the elliptic equation. It is easy to see that $u(t, x)=(\exp (-t)+t+1)(\cos (x)-1)$ and $p(x)=$ $3 \cos (x)+\cos (2 x)-1$ are the exact solutions of (4.1).

For this example, we have the following auxiliary nonlocal boundary value problem for an unknown function $v(t, x)$ :

$$
\left\{\begin{array}{l}
-\frac{d^{2} v(t, x)}{d t^{2}}-\frac{\partial}{\partial x}\left((2+\cos x) \frac{\partial v(t, x)}{\partial x}\right)+v(t, x)=f(t, x), \quad 0<x<\pi, 0<t<T, \\
v(t, 0)=v_{x}(t, \pi)=0, \quad 0 \leq t \leq T, \\
v(0, x)-v(\lambda, x)=(1-\exp (-\lambda)-\lambda)(\cos (x)-1), \quad 0 \leq x \leq \pi, \\
v(T, x)-v(\lambda, x)=(\exp (-T)-\exp (-\lambda)+T-\lambda)(\cos (x)-1), \quad 0 \leq x \leq \pi .
\end{array}\right.
$$

Consider the set $[0, T]_{\tau} \times[0, \pi]_{h}$ of grid points depending on the small parameters $\tau$ and $h$,

$$
\begin{aligned}
{[0, T]_{\tau} \times[0, \pi]_{h}=} & \left\{\left(t_{k}, x_{n}\right): t_{k}=k \tau, k=1, \ldots, N-1, N \tau=T,\right. \\
& \left.x_{n}=n h, n=1, \ldots, M-1, M h=\pi\right\} .
\end{aligned}
$$

We get difference schemes of the first order of accuracy in $t$ and the second order of accuracy in $x$,

$$
\left\{\begin{array}{l}
\frac{v_{n}^{k+1}-2 v_{n}^{k}+v_{n}^{k-1}}{\tau^{2}}+\left(2+\cos \left(x_{n}\right)\right) \frac{v_{n+1}^{k}-2 v_{n}^{k}+v_{n-1}^{k}}{h^{2}}-\sin \left(x_{n}\right) \frac{v_{n+1}^{k}-v_{n-1}^{k}}{2 h}-v_{n}^{k}=\theta_{n}^{k} \\
\theta_{n}^{k}=-f\left(t_{k}, x_{n}\right), k=1, \ldots, N-1, n=1, \ldots, M-1, \\
v_{0}^{k}=0, \quad v_{M}^{k}-v_{M-1}^{k}=0, \quad k=0, \ldots, N, \\
v_{n}^{0}-v_{n}^{l}=(1-\exp (-\lambda)-\lambda)\left(\cos \left(x_{n}\right)-1\right), \quad n=0, \ldots, M, \\
v_{n}^{N}-v_{n}^{l}=\left(\exp \left(-t_{N}\right)-\exp (-\lambda)+t_{N}-\lambda\right)\left(\cos \left(x_{n}\right)-1\right), \\
\quad n=0, \ldots, M, l=\left[\frac{\lambda}{\tau}\right]
\end{array}\right.
$$

for the approximate solutions of the nonlocal boundary value problem (4.2). 
Now, it is easy to rewrite difference scheme (4.3) in the following matrix form:

$$
\begin{aligned}
& A_{n} v_{n+1}+B_{n} v_{n}+C_{n} v_{n-1}=I_{N+1} \theta_{n}, \quad n=1, \ldots, M-1, \\
& v_{0}=\overrightarrow{0}, \quad v_{M}=v_{M-1} .
\end{aligned}
$$

Here, $\theta_{n}$ is an $(N+1) \times 1$ column matrix, $I_{N+1}$ is the $(N+1) \times(N+1)$ identity matrix, $A_{n}$, $B_{n}, C_{n}$ are $(N+1) \times(N+1)$ square matrices

$$
\begin{aligned}
& A_{n}=\left[\begin{array}{ccccccc}
0 & 0 & 0 & \cdots & 0 & 0 & 0 \\
0 & a_{n} & 0 & \cdots & 0 & 0 & 0 \\
0 & 0 & a_{n} & \cdots & 0 & 0 & 0 \\
\vdots & \vdots & \vdots & \cdots & \vdots & \vdots & \vdots \\
0 & 0 & 0 & \cdots & a_{n} & 0 & 0 \\
0 & 0 & 0 & \cdots & 0 & a_{n} & 0 \\
0 & 0 & 0 & \cdots & 0 & 0 & 0
\end{array}\right] \\
& B_{n}=\left[\begin{array}{ccccccccccc}
1 & 0 & 0 & 0 & \cdots & -1 & \cdots & 0 & 0 & 0 & 0 \\
d & b_{n} & d & 0 & \cdots & 0 & \cdots & 0 & 0 & 0 & 0 \\
0 & d & b_{n} & d & \cdots & 0 & \cdots & 0 & 0 & 0 & 0 \\
\vdots & \vdots & \vdots & \vdots & \vdots & \vdots & \cdots & \vdots & \vdots & \vdots & \vdots \\
0 & 0 & 0 & 0 & \cdots & 0 & \cdots & d & b_{n} & d & 0 \\
0 & 0 & 0 & 0 & \cdots & 0 & \cdots & 0 & d & b_{n} & d \\
0 & 0 & 0 & 0 & \cdots & -1 & \cdots & 0 & 0 & 0 & 1
\end{array}\right] \text {, } \\
& C_{n}=\left[\begin{array}{ccccccc}
0 & 0 & 0 & \cdots & 0 & 0 & 0 \\
0 & c_{n} & 0 & \cdots & 0 & 0 & 0 \\
0 & 0 & c_{n} & \cdots & 0 & 0 & 0 \\
\vdots & \vdots & \vdots & \cdots & \vdots & \vdots & \vdots \\
0 & 0 & 0 & \cdots & c_{n} & 0 & 0 \\
0 & 0 & 0 & \cdots & 0 & c_{n} & 0 \\
0 & 0 & 0 & \cdots & 0 & 0 & 0
\end{array}\right] \\
& a_{n}=\frac{2+\cos \left(x_{n}\right)}{h^{2}}-\frac{\sin \left(x_{n}\right)}{2 h}, \quad b_{n}=-\frac{2}{\tau^{2}}-\frac{2\left(2+\cos \left(x_{n}\right)\right)}{h^{2}}-1, \\
& c_{n}=\frac{2+\cos \left(x_{n}\right)}{h^{2}}+\frac{\sin \left(x_{n}\right)}{2 h}, \quad d=\frac{1}{\tau^{2}}, \\
& \theta_{n}=\left[\begin{array}{c}
\theta_{n}^{0} \\
\vdots \\
\theta_{n}^{N}
\end{array}\right] \\
& \theta_{n}^{k}=-f\left(t_{k}, x_{n}\right), \quad k=1, \ldots, N-1, n=1, \ldots, M-1, \\
& \theta_{n}^{0}=(1-\exp (-\lambda)-\lambda)\left(\cos \left(x_{n}\right)-1\right) \text {, } \\
& \theta_{n}^{N}=\left(\exp \left(-t_{N}\right)-\exp (-\lambda)+t_{N}-\lambda\right)\left(\cos \left(x_{n}\right)-1\right), \quad n=1, \ldots, M-1 \text {, } \\
& v_{n-1}=\left[\begin{array}{c}
v_{n-1}^{0} \\
\vdots \\
v_{n-1}^{N}
\end{array}\right], \quad v_{n}=\left[\begin{array}{c}
v_{n}^{0} \\
\vdots \\
v_{n}^{N}
\end{array}\right], \quad v_{n+1}=\left[\begin{array}{c}
v_{n+1}^{0} \\
\vdots \\
v_{n+1}^{N}
\end{array}\right] \text {. }
\end{aligned}
$$


For numerical solving of this system equation, we use the modified Gauss elimination method [31]. Solution of (4.4) is presented by the formula

$$
v_{n}=\alpha_{n+1} v_{n+1}+\beta_{n+1}, \quad n=M-1, \ldots, 1,
$$

where $\alpha_{n}(n=1, \ldots, M-1)$ are $(N+1) \times(N+1)$ square matrices and $\beta_{n}(n=1, \ldots, M-1)$ are $(N+1) \times 1$ column matrices. For $\alpha_{n+1}, \beta_{n+1}$, we have the following formulas:

$$
\begin{aligned}
& \alpha_{n+1}=-\left(B_{n}+C_{n} \alpha_{n}\right)^{-1} A_{n}, \\
& \beta_{n+1}=\left(B_{n}+C_{n} \alpha_{n}\right)^{-1}\left(I_{N+1} \theta_{n}-C_{n} \beta_{n}\right), \quad n=1, \ldots, M-1,
\end{aligned}
$$

where

$$
\begin{aligned}
& \alpha_{1}=0_{(N+1) \times(N+1)}, \quad \beta_{1}=0_{(N+1) \times 1} \quad\left(v_{0}=\overrightarrow{0}\right), \\
& \alpha_{M}=I_{N+1}, \quad \beta_{M}=0_{(N+1) \times 1} \quad\left(v_{M-1}=v_{M}\right) .
\end{aligned}
$$

By using the second order of accuracy in $x$ approximation of $A$, we get the following values of $p$ in grid points:

$$
\begin{aligned}
p_{n}= & -\frac{\left(2+\cos \left(x_{n}\right)\right)}{h^{2}}\left[\left(\varphi_{n+1}-v_{n+1}^{0}\right)-2\left(\varphi_{n}-v_{n}^{0}\right)+\left(\varphi_{n-1}-v_{n-1}^{0}\right)\right] \\
& +\frac{\sin \left(x_{n}\right)}{2 h}\left(\left(\varphi_{n+1}-v_{n+1}^{0}\right)-\left(\varphi_{n-1}-v_{n-1}^{0}\right)\right)+\left(\varphi_{n}-v_{n}^{0}\right), \\
& n=1, \ldots, M-1 .
\end{aligned}
$$

For the approximate solution of problem (4.1), we use the formula

$$
u_{n}^{k}=v_{n}^{k}+\varphi_{n}-v_{n}^{0}, \quad n=0, \ldots, M, k=0, \ldots, N
$$

Now, we consider again nonlocal boundary value problem (4.2).

Applying (3.7), we get the second order of accuracy in $t$ and $x$ difference scheme

$$
\left\{\begin{array}{l}
\frac{v_{n}^{k+1}-2 v_{n}^{k}+v_{n}^{k-1}}{\tau^{2}}+\left(2+\cos \left(x_{n}\right)\right) \frac{v_{n+1}^{k}-2 v_{n}^{k}+v_{n-1}^{k}}{h^{2}} \\
\quad-\sin \left(x_{n}\right) \frac{v_{n+1}^{k}-v_{n-1}^{k}}{2 h}-v_{n}^{k}=\theta_{n}^{k}, \\
\quad \theta_{n}^{k}=-f\left(t_{k}, x_{n}\right), k=1, \ldots, N-1, n=1, \ldots, M-1, \\
v_{0}^{k}=0, \quad-3 v_{M}^{k}+4 v_{M-1}^{k}-v_{M-2}^{k}=0, \quad k=0, \ldots, N, \\
10 v_{M}^{k}-15 v_{M-1}^{k}+6 v_{M-2}^{k}-v_{M-3}^{k}=0, \\
v_{n}^{0}+\left(\frac{\lambda}{\tau}-l-1\right) v_{n}^{l}-\left(\frac{\lambda}{\tau}-l\right) v_{n}^{l+1}=(1-\exp (-\lambda)-\lambda) \cos \left(x_{n}\right), \\
\quad n=0, \ldots, M, \\
v_{n}^{N}+\left(\frac{\lambda}{\tau}-l-1\right) v_{n}^{l}-\left(\frac{\lambda}{\tau}-l\right) v_{n}^{l+1} \\
\quad=\left(\exp \left(-t_{N}\right)-\exp (-\lambda)+t_{N}-\lambda\right) \cos \left(x_{n}\right), \quad n=0, \ldots, M
\end{array}\right.
$$

for the approximate solution of nonlocal boundary value problem (4.2). 
Therefore, we can rewrite difference scheme (4.9) in the matrix form

$$
\begin{aligned}
& A_{n} v_{n+1}+B_{n} v_{n}+C_{n} v_{n-1}=I_{N+1} \theta_{n}, \quad n=1, \ldots, M-1, \\
& v_{0}=0, \quad-3 v_{M}+4 v_{M-1}-v_{M-2}=0,
\end{aligned}
$$

where $A_{n}, C_{n}$ are defined by (4.5), (4.6), (4.7) and $B_{n}$ is an $(N+1) \times(N+1)$ square matrix

$$
\begin{aligned}
& B_{n}=\left[\begin{array}{cccccccccccccc}
1 & 0 & 0 & 0 & \cdots & 0 & y & z & 0 & \cdots & 0 & 0 & 0 & 0 \\
d & b_{n} & d & 0 & \cdots & 0 & 0 & 0 & 0 & \cdots & 0 & 0 & 0 & 0 \\
0 & d & b_{n} & d & \cdots & 0 & 0 & 0 & 0 & \cdots & 0 & 0 & 0 & 0 \\
0 & 0 & d & b_{n} & \cdots & 0 & 0 & 0 & 0 & \cdots & 0 & 0 & 0 & 0 \\
\vdots & \vdots & \vdots & \vdots & \vdots & \vdots & \vdots & \vdots & \vdots & \vdots & \vdots & \vdots & \vdots & \vdots \\
0 & 0 & 0 & 0 & \cdots & 0 & 0 & 0 & 0 & \cdots & b_{n} & d & 0 & 0 \\
0 & 0 & 0 & 0 & \cdots & 0 & 0 & 0 & 0 & \cdots & d & b_{n} & d & 0 \\
0 & 0 & 0 & 0 & \cdots & 0 & 0 & 0 & 0 & \cdots & 0 & d & b_{n} & d \\
0 & 0 & 0 & 0 & \cdots & 0 & y & z & 0 & \cdots & 0 & 0 & 0 & 1
\end{array}\right] \\
& b_{n}=-\frac{2}{\tau^{2}}-\frac{2\left(2+\cos \left(x_{n}\right)\right)}{h^{2}}-1, \quad d=\frac{1}{\tau^{2}}, \quad y=\left(\frac{\lambda}{\tau}-l-1\right), \quad z=-\left(\frac{\lambda}{\tau}-l\right) \text {. }
\end{aligned}
$$

For solution of (4.10), we use the formula

$$
v_{n}=\alpha_{n} v_{n+1}+\beta_{n} v_{n+2}+\gamma_{n}, \quad n=M-2, \ldots, 0,
$$

where $\alpha_{n}, \beta_{n}(n=0, \ldots, M-2)$ are $(N+1) \times(N+1)$ square matrices and $\gamma_{n}(n=0, \ldots, M-2)$ are $(N+1) \times 1$ column matrices. For the coefficients $\alpha_{n}, \beta_{n}, \gamma_{n}$, we get formulas

$$
\begin{aligned}
& \alpha_{n}=-\left(B_{n}+C_{n} \alpha_{n-1}\right)^{-1}\left(A_{n}+C_{n} \beta_{n-1}\right), \\
& \beta_{n}=0, \\
& \gamma_{n}=\left(B_{n}+C_{n} \alpha_{n-1}\right)^{-1}\left(I_{N+1} \theta_{n}-C_{n} \gamma_{n-1}\right), \quad n=1, \ldots, M-1 .
\end{aligned}
$$

Here

$$
\begin{aligned}
& \alpha_{0}=0, \quad \beta_{0}=0, \quad \gamma_{0}=0 \quad\left(v_{0}=0\right), \\
& \alpha_{1}=\frac{8}{5} I_{N+1}, \quad \beta_{1}=-\frac{3}{5} I_{N+1}, \\
& \alpha_{M-2}=4 I_{N+1}, \quad \beta_{M-2}=-3 I_{N+1}, \\
& \alpha_{M-3}=\frac{8}{3} I_{N+1}, \quad \beta_{M-3}=-\frac{5}{3} I_{N+1},
\end{aligned}
$$

and $\gamma_{0}, \gamma_{1}, \gamma_{M-2}, \gamma_{M-3}$ are the $(N+1) \times 1$ zero column vector. For $v_{M}$ and $v_{M-1}$, we have the following formulas:

$$
\begin{aligned}
& v_{M}=\left(Q_{11}-Q_{12} Q_{22}^{-1} Q_{21}\right)^{-1}\left(G_{1}-Q_{12} Q_{22}^{-1} G_{2}\right), \\
& v_{M-1}=Q_{22}^{-1}\left(G_{2}-Q_{21} v_{M}\right),
\end{aligned}
$$


where

$$
\begin{aligned}
& Q_{11}=-3 A_{M-2}-8 B_{M-2}-8 C_{M-2} \alpha_{M-3}-3 C_{M-2} \beta_{M-3}, \\
& Q_{12}=4 A_{M-2}+9 B_{M-2}+9 C_{M-2} \alpha_{M-3}+4 C_{M-2} \beta_{M-3}, \\
& Q_{21}=-3 B_{M-1}-8 C_{M-1}, \\
& Q_{22}=A_{M-1}+4 B_{M-1}+9 C_{M-1}, \\
& G_{1}=I_{N+1} \theta_{M-2}-C_{M-2} \gamma_{M-3}, \\
& G_{2}=I_{N+1} \theta_{M-1} .
\end{aligned}
$$

Now, by using MATLAB programs, we present numerical results for this example (4.1). The numerical solutions are recorded for different values of $N$ and $M$.

The numerical solutions are recorded for different values of $N=M$ for $T=2$. The grid function $u_{n}^{k}$ represents the numerical solution of difference schemes for inverse problem at $\left(t_{k}, x_{n}\right)$, respectively. Grid function $p_{n}$ represents the numerical solutions at $x_{n}$ for unknown function $p$. For their comparison, the errors are computed by

$$
\begin{aligned}
& \mathrm{E} u_{M}^{N}=\max _{1 \leq k \leq N-1}\left(\sum_{n=1}^{M-1}\left|u\left(t_{k}, x_{n}\right)-u_{n}^{k}\right|^{2} h\right)^{\frac{1}{2}}, \\
& \mathrm{E} p_{M}=\left(\sum_{n=1}^{M-1}\left|p\left(x_{n}\right)-p_{n}\right|^{2} h\right)^{\frac{1}{2}} .
\end{aligned}
$$

Tables 1 and 2 give the error analysis between the exact solution and solutions derived by difference schemes. Tables 1 and 2 are constructed for $N=M=20,40,80$, and 160 . Hence, the second order of accuracy difference scheme is more accurate comparing with the first order of accuracy difference scheme.

Table 1 is the error analysis between the exact solution $p$ and solutions derived by difference schemes in the second stage of algorithm.

Table 2 presents the error analysis between the exact solution $u$ and solutions derived by first order and second order accuracy of difference schemes.

Table 1 Error analysis for $p$

\begin{tabular}{lllll}
\hline & $\mathbf{N}=\boldsymbol{M}=\mathbf{2 0}$ & $\mathbf{N}=\mathbf{M}=\mathbf{4 0}$ & $\mathbf{N}=\boldsymbol{M}=\mathbf{8 0}$ & $\mathbf{N =} \boldsymbol{M = 1 6 0}$ \\
\hline $\begin{array}{l}\text { First order of accuracy } \\
\text { difference scheme }\end{array}$ & 0.25111 & 0.24195 & 0.11044 & 0.045366 \\
$\begin{array}{l}\text { Second order of accuracy } \\
\text { difference scheme }\end{array}$ & 0.024061 & 0.0052977 & 0.0012825 & $3.16 \times 10^{-4}$ \\
\hline
\end{tabular}

Table 2 Error analysis for $u$

\begin{tabular}{lllll}
\hline & $\mathbf{N}=\boldsymbol{M}=\mathbf{2 0}$ & $\mathbf{N}=\mathbf{M}=\mathbf{4 0}$ & $\mathbf{N}=\boldsymbol{M}=\mathbf{8 0}$ & $\boldsymbol{N}=\boldsymbol{M}=\mathbf{1 6 0}$ \\
\hline $\begin{array}{l}\text { First order of accuracy } \\
\text { difference scheme }\end{array}$ & 0.036174 & 0.042733 & 0.016978 & 0.0041271 \\
$\begin{array}{l}\text { Second order of accuracy } \\
\text { difference scheme }\end{array}$ & 0.019509 & 0.0034552 & $6.41 \times 10^{-4}$ & $1.56 \times 10^{-4}$ \\
\hline
\end{tabular}




\section{Conclusion}

In this paper, the inverse problem for a multidimensional elliptic equation with DirichletNeumann conditions and overdetermination is considered. The first and second orders of accuracy difference schemes for approximate solution of this problem are presented. Theorems on the stability, almost coercive stability, and coercive stability inequalities for the solutions of difference schemes for inverse problem are proved. Numerical example in a two-dimensional case is given. As it can be seen from Tables 1 and 2, the second order of accuracy difference scheme is more accurate comparing with the first order of accuracy difference scheme.

\section{Competing interests}

The authors declare that they have no competing interests.

\section{Authors' contributions}

All authors contributed equally to the writing of this paper. All authors read and approved the final manuscript.

\section{Author details}

'Department of Mathematical Engineering, Gumushane University, Gumushane, 29100, Turkey. ${ }^{2}$ TAU, Ashgabat, Turkmenistan.

Received: 27 November 2014 Accepted: 6 March 2015 Published online: 17 March 2015

\section{References}

1. Samarskii, AA, Vabishchevich, PN: Numerical Methods for Solving Inverse Problems of Mathematical Physics. Inverse and III-Posed Problems Series. de Gruyter, Berlin (2007)

2. Prilepko, Al, Orlovsky, DG, Vasin, IA: Methods for Solving Inverse Problems in Mathematical Physics. Dekker, New York (2000)

3. Kabanikhin, SI: Inverse and III-Posed Problems: Theory and Applications. de Gruyter, Berlin (2011)

4. Orlovskii, DG: Inverse Dirichlet problem for an equation of elliptic type. Differ. Equ. 44(1), 124-134 (2008)

5. Orlovsky, DG, Piskarev, S: On approximation of inverse problems for abstract elliptic problems. J. Inverse III-Posed Probl. 17(8), 765-782 (2009)

6. Orlovsky, DG, Piskarev, S: The approximation of Bitzadze-Samarsky type inverse problem for elliptic equations with Neumann conditions. Contemp. Anal. Appl. Math. 1(2), 118-131 (2013)

7. Soloviev, W: Inverse problems of source determination for the two-dimensional Poisson equation. Zh. Vychisl. Mat. Mat. Fiz. 44(5), 862-871 (2004)

8. Soloviev, W: Inverse problems for elliptic equations on the plane I. Differ. Equ. 42(8), 1106-1114 (2006)

9. Soloviev, $\mathrm{W}$ : Inverse problems of determining the source and coefficient in an elliptic equation in a rectangle. Zh. Vychisl. Mat. Mat. Fiz. 47(8), 1365-1377 (2007)

10. Soloviev, WV: Inverse coefficient problems for elliptic equations in a cylinder: I. Differ. Equ. 49(7), 908-916 (2011)

11. Soloviev, WV: Inverse coefficient problems for elliptic equations in a cylinder: II. Differ. Equ. 49(8), 996-1005 (2011)

12. Ashyralyev, A, Erdogan, AS: Well-posedness of the inverse problem of a multidimensional parabolic equation. Vestn. Odessa Nat. Univ., Math. Mech. 15(18), 129-135 (2010)

13. Ashyralyev, A: On the problem of determining the parameter of a parabolic equation. Ukr. Math. J. 62(9), 1397-1408 (2011)

14. Ashyralyev, A, Erdogan, AS, Demirdag, O: On the determination of the right-hand side in a parabolic equation. Appl. Numer. Math. 62(11), 1672-1683 (2012)

15. Ashyralyyev, C, Dural, A, Sozen, Y: Finite difference method for the reverse parabolic problem. Abstr. Appl. Anal. 2012 Article ID 294154 (2012)

16. Ashyralyyev, C, Demirdag, O: The difference problem of obtaining the parameter of a parabolic equation. Abstr. Appl. Anal. 2012, Article ID 603018 (2012)

17. Ashyralyyev, C, Dural, A, Sozen, Y: Finite difference method for the reverse parabolic problem with Neumann condition. In: Ashyralyev, A, Lukasov, A (eds.) First International Conference on Analysis and Applied Mathematics (ICAAM 2012). AIP Conference Proceedings, vol. 1470, pp. 102-105 (2012)

18. Ashyralyyev, C, Dedeturk, M: Finite difference method for the inverse elliptic problem with Dirichlet condition. Contemp. Anal. Appl. Math. 1(2), 132-155 (2013)

19. Ashyralyyev, C, Dedeturk, M: Approximate solution of inverse problem for elliptic equation with overdetermination. Abstr. Appl. Anal. 2013, Article ID 548017 (2013)

20. Ashyralyev, A, Urun, M: Determination of a control parameter for the Schrödinger equation. Contemp. Anal. Appl. Math. 1(2), 156-166 (2013)

21. Ashyralyyev, C: High order of accuracy difference schemes for the inverse elliptic problem with Dirichlet condition. Bound. Value Probl. 2014, 5 (2014). doi:10.1186/1687-2770-2014-5

22. Ashyralyev, A, Ashyralyyev, C: On the problem of determining the parameter of an elliptic equation in a Banach space. Nonlinear Anal., Model. Control 19(3), 350-366 (2014)

23. Ashyralyyev, C, Dedeturk, M: Approximation of the inverse elliptic problem with mixed boundary value conditions. In: Ashyralyev, A, Malkowsky, E (eds.) International Conference on Analysis and Applied Mathematics (ICAAM 2014). AIP Conference Proceedings, vol. 1611, pp. 25-29 (2014) 
24. Ashyralyyev, C: Inverse Neumann problem for an equation of elliptic type. In: Ashyralyev, A, Malkowsky, E (eds.) International Conference on Analysis and Applied Mathematics (ICAAM 2014). AIP Conference Proceedings, vol. 1611, pp. 46-52 (2014)

25. Ashyralyyev, C: High order approximation of the inverse elliptic problem with Dirichlet-Neumann conditions. Filomat 28(5), 947-962 (2014). doi:10.2298/FIL1405947A

26. Krein, SG: Linear Differential Equations in Banach Space. Nauka, Moscow (1966)

27. Ashyralyev, A, Sobolevskii, PE: New Difference Schemes for Partial Differential Equations. Operator Theory: Advances and Applications. Birkhäuser, Basel (2004)

28. Ashyralyev, A, Ozturk, E: The numerical solution of the Bitsadze-Samarskii nonlocal boundary value problems with the Dirichlet-Neumann condition. Abstr. Appl. Anal. 2012, Article ID 730804 (2012)

29. Ashyralyev, A, Ozesenli Tetikoglu, FS: FDM for elliptic equations with Bitsadze-Samarskii-Dirichlet conditions. Abstr. Appl. Anal. 2012, Article ID 454831 (2012)

30. Sobolevskii, PE: Difference Methods for the Approximate Solution of Differential Equations. Voronezh State University Press, Voronezh (1975)

31. Samarskii, AA, Nikolaev, ES: Numerical Methods for Grid Equations, vol. 2. Birkhäuser, Basel (1989)

Submit your manuscript to a SpringerOpen ${ }^{\circ}$ journal and benefit from:

- Convenient online submission

Rigorous peer review

- Immediate publication on acceptance

- Open access: articles freely available online

- High visibility within the field

- Retaining the copyright to your article 